\title{
Adjacent Segment Degeneration after Single-Level PLIF: Comparison between Spondylolytic Spondylolisthesis, Degenerative Spondylolisthesis and Spinal Stenosis
}

\author{
Chang Hun Yu, Jung Eun Lee, Jae Jun Yang, Bong-Soon Chang, Choon-Ki Lee \\ Department of Orthopaedic Surgery, Seoul National University College of Medicine, Seoul, Korea
}

Study Design: A retrospective study.

Purpose: To comparatively investigated the rate of the adjacent segment degeneration and the clinical outcomes in patients with spondylolytic spondylolisthesis, spinal stenosis or degenerative spondylolisthesis.

Overview of Literature: There have been few studies reported on the adjacent segment degeneration following posterior lumbar interbody fusion(PLIF). Many risk factors for the adjacent segment degeneration following PLIF have been proposed. The range of decompression has been presented as one of the risk factors, yet controversial.

Methods: This study enrolled sixty-three patients who had been treated with single-level PLIF and who were followed up for more than two years. The patients were divided into 3 groups based on the preoperative diagnosis. We analyzed the dif ference between the preoperative and postoperative intervertebral disc heights of the superior adjacent segments. The incidence rates of instability and the clinical outcomes were comparatively analyzed between each group.

Results: The average age of the patients was 55.8 years in the spondylolytic spondylolisthesis group, 65.9 years in the degenerative spondylolisthesis group and 60.4 years in the spinal stenosis group. The average follow-up period was 44 months, 43 months and 42 months, respectively. At the last follow-up, compared to the preoperative period, the intervertebral disc height decreased in all three groups. A statistically significant decrease $(p<0.01)$ was observed only in the spondylolytic spondylolisthesis group and no significant difference was observed between each group ( $p=0.41$ ). The incidence rate of instability and the clinical outcome were not significantly different between each group.

Conclusions: Spondylolytic spondylolisthesis with total laminectomy and single-level PLIF showed no significant difference in the superior adjacent segment degeneration and instability, and the clinical outcome as compared to that of partial laminectomy with single-level PLIF for treating degenerative spondylolisthesis or spinal stenosis.

Key Words: Spondylolytic spondylolisthesis, Adjacent segment disease, Posterior lumbar interbody fusion

\section{Introduction}

As a vertebral body becomes anteriority displaced due to an isthmus defect, spondylolytic spondylolisthesis causes mechanically induced low back pain or it compresses the neural structures and causes neurological symptoms. Surgical management is performed for the patients who are refractory to the conservative management or for those who present with progressive neurological symptoms. Using a

Received Apr 12, 2010; 1st Revised Jun 1, 2010; Accepted Jun 17, 2010

Corresponding author: Bong-Soon Chang, MD

Department of Orthopaedic Surgery, Seoul National University Hospital, Seoul National University College of Medicine,

28 Yeongeon-dong, Jongno-gu, Seoul 110-744, Korea

Tel: +82-2-2072-3864, Fax: +82-2-764-2718, E-mail: bschang@ snu.ac.kr 
pedicle screw and a cage following the decompression, posterior lumbar interbody fusion (PLIF) has been performed as one of the conventional treatments [1]. A higher fusion rate can be obtained in cases in which rigid fixation is performed using a pedicle screw, as compared with that for the cases in which internal fixation was not performed, and the postoperative period of using a brace can be shortened with rigid fixation using a pedicle screw. This is advantageous in that ambulation can be more quickly performed without using a brace [2,3]. PLIF has been reported to produce good clinical outcomes. However, it has also been reported to produce complications due to the rigid fixation. Of these complications, adjacent segment disease has been a matter of increasing interest.

In cases in which solid fusion is achieved, the mobility of the superior adjacent segment is increased and a loading is increasingly exerted to the superior adjacent disc. This has been verified in a cadaver study, and it has also been shown in a biomechanical study $[4,5]$. But there are also opinions that this is all part of the normal aging or degenerative process [6-8]. The reported risk factors for adjacent segment disease are old age, long level fusion, the degenerative changes in the adjacent segment that are detected before the surgery, the facet joint sagittalization and the laminar inclination [8-12]. Another report says that the instability of the superior adjacent segment is increased with posterolateral fusion after removing the laminar, spinous process and the posterior ligament complex of the superior adjacent segment [13]. In cases in which a decompression is performed for spondylolytic spondylolisthesis, the total excision of the laminar and spinous process of fusion level and the posterior ligament complex of the superior adjacent segment is unavoidable. Therefore, we concerned about that the rate of early degenerative change at the superior adjacent segment is relatively high.

Given the above background, we examined whether the degenerative changes of the adjacent segment occurred in the early stage following a single-level PLIF after the decompression of the spondylolytic spondylolisthesis and if this results in poor clinical outcomes. To do this, we compared the adjacent segment degeneration and clinical outcomes between the cases of spondylolytic spondylolisthesis and those cases of spinal stenosis and degenerative spondylolisthesis that were treated with partial laminectomy and a single-level PLIF.

\section{Materials and Methods}

\section{Study patients}

The current study was conducted on the patients who could be followed up for a minimum period of two years following a single-level posterior decompression and the PLIF with using a cage for the management of lumbar disease that was refractory to conservative management at our medical institution from May 2000 to July 2007. The cases of non-union at the fusion level, the cases requiring revision and the cases with existing instability in the superior adjacent segment were excluded from the analysis. A total of 63 patients were enrolled and their average age was $61.1 \pm$ 10.1 years (range, 29 to 78 years). There were 14 men $(22.2 \%)$ and 49 women $(77.8 \%)$. The average follow-up period was $43.1 \pm 18.6$ months (range, 24 to 102 months). Based on the preoperative diagnosis, the patients were classified into the spondylolytic spondylolisthesis group $(\mathrm{n}=$ $17,27.0 \%)$, the degenerative lumbar spondylolisthesis group $(n=22,34.9 \%)$ and the spinal stenosis group $(n=24$, $38.1 \%)$.

The surgeries of three groups were performed by two spine surgeons among the authors. The target site was reached via a subperiosteal dissection after making a midline skin incision. At the corresponding fusion level, a pedicle screw was inserted and this was followed by a decompression procedure. In regard to the amount of decompression, in the degenerative lumbar spondylolisthesis group and the spinal stenosis group, partial laminectomy and the removal of the posterior ligament complex (the interspinous ligament and the supraspinous ligament) and the ligament flavum were performed at the fusion level. In the spondylolytic spondylolisthesis group, total lamenectomy was inevitably performed. According to this, the posterior ligament complex and ligament flavum were removed at the fusion level and the superior level.

\section{Assessment and analysis methods}

To confirm the presence of degenerative changes of the superior adjacent disc, we measured the intervetebral disc height of the superior adjacent segment using the standing lateral plain radiography that was taken at preoperation and at the time of the last follow up. To confirm the occurrence of instability, we measured the superior adjacent disc seg- 


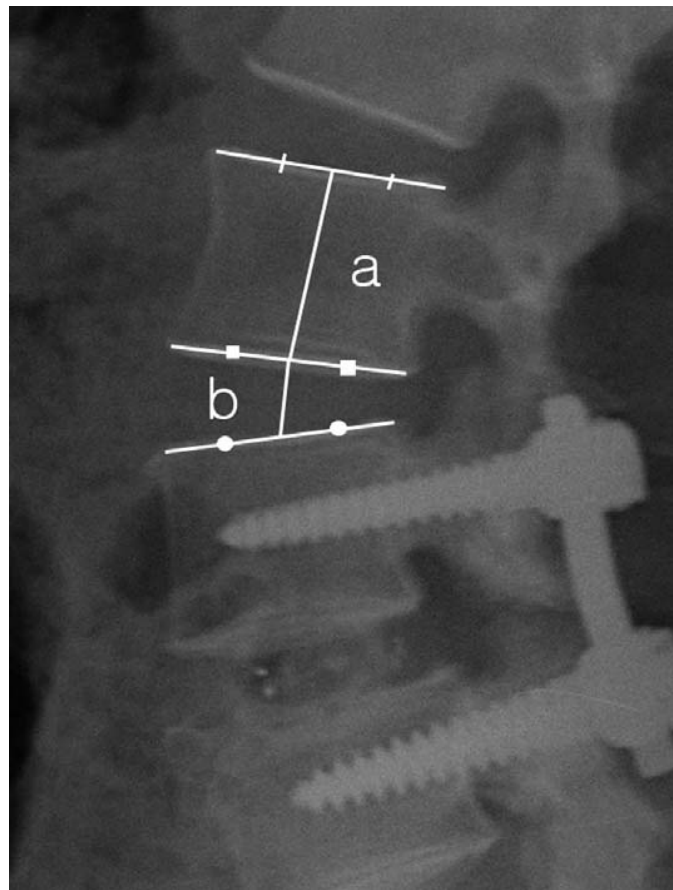

Fig. 1. Measurement technique for the intervertebral disc height ratio (b/a). The vertebral body height (a) is defined as the distance between the mid-point of the upper and lower end plates of the vertebral body. The intervertebral disc height (b) is defined as the distance between the mid-point of the lower end plate of the upper vertebral body and the upper end plate of the lower vertebral body. mental sagittal angle [14] and the occurrence of anterior or posterior listhesis. The intervertebral disc height was evaluated using a PACS viewer (M-view, Seoul, Korea) by measuring the distance extending from the mid-point on the upper end-plate of the superior vertebral body at the fusion level to the mid-point on the lower end-plate of the superior vertebral body in the superior adjacent segment. To minimize the errors due to a magnified ratio on the radiographic images, we compared the ratio between the intervertebral disc height and the body height of the superior vertebral body in the superior adjacent segment (Fig. 1). At preoperation and at the last follow up, the segmental sagittal angle of the superior adjacent segment was defined as the angle formed between the upper end-plate and the lower end-plate of the superior adjacent level on a standing lateral flexion and extension views, and the angle was measured using Cobb's method (Fig. 2). In cases in which there was a discrepancy in the angle of $>10^{\circ}$, it was determined that hypermobility was present. The occurrence of anterior or posterior listhesis was determined to be positive when the standing lateral plain radiography at the last follow up shows a listhesis distance (Fig. 3) of more than $3 \mathrm{~mm}$ compared to that of the preoperation image. For cases in which the segmental sagittal angle showed hypermobility or listhesis, it was determined that instability had occurred. Any
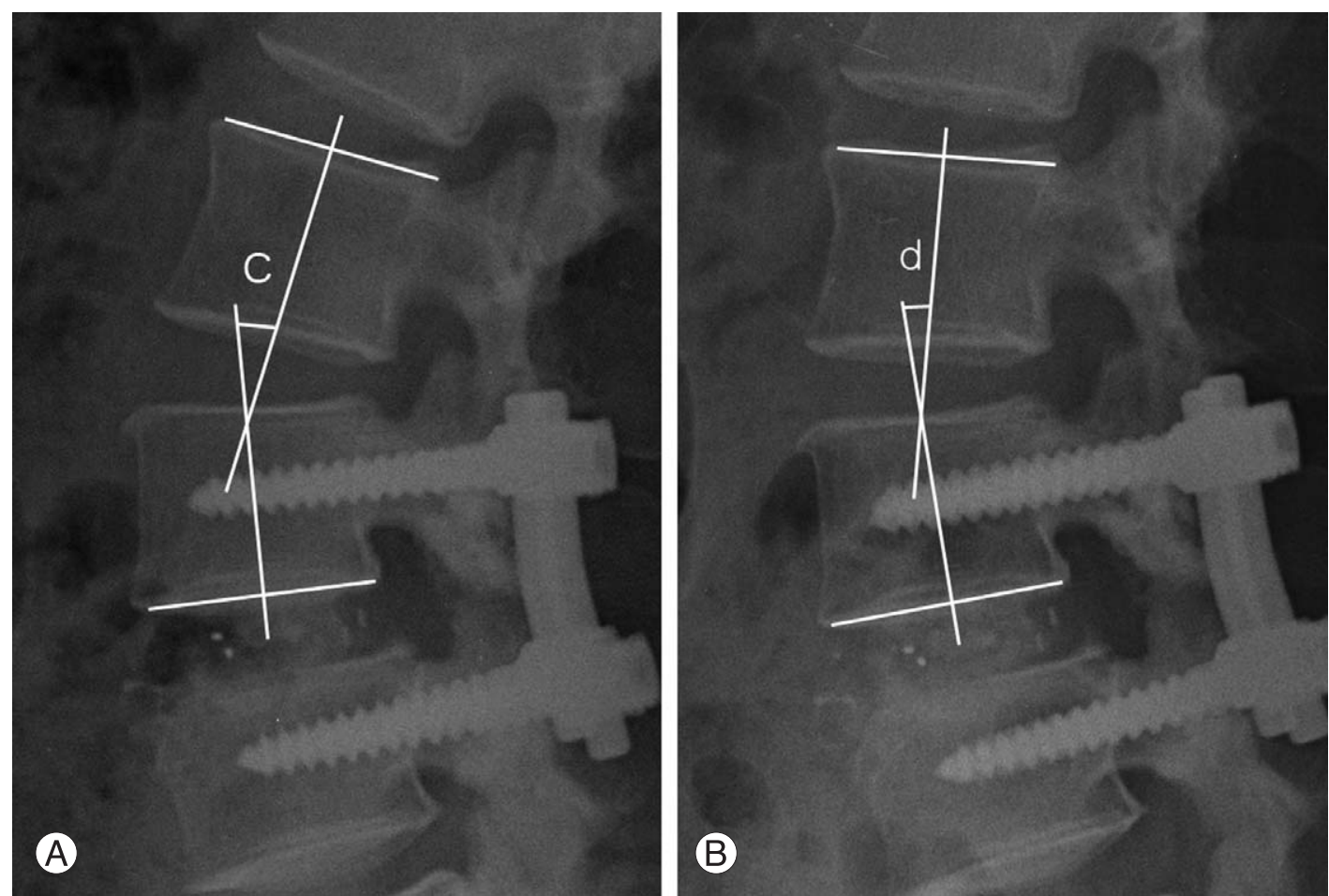

Fig. 2. Measurement technique for the segmental sagittal angle (c, d). This was measured by Cobb's method at the upper segment of the fused segment in the extension position (A) and flexion position (B). 
cases in which both hypermobility and listhesis occurred were considered to be a single case. At a last follow-up, the clinical outcomes were evaluated using Brodsky's method (Table 1) [15].

Each parameter was measured twice by two orthopedic surgeons. We used SPSS ver. 17 (SPSS Inc., Chicago, IL, USA). A comparison between the intraobserver and interobserver variations for the measured superior adjacent segment's preoperative body-disc height ratio/last body-disc height ratio and the segmental sagittal angles at the last fol-

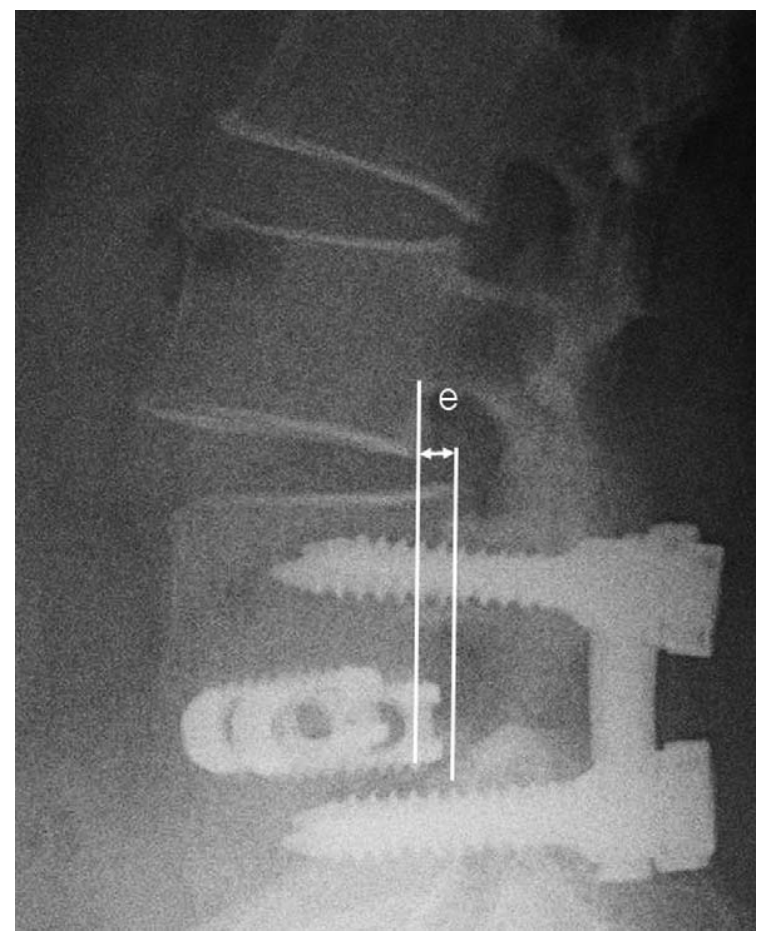

Fig. 3. Measurement technique for the listhesis distance (e). The listhesis distance is defined as the distance between the posterior-lower portion of the upper vertebral body and the posterior border of the lower vertebral body. low up were assessed using Spearman's correlation analysis. A comparison of the clinical outcomes was done using kappa values. Furthermore, inter-group comparison was done using the average value of two measurements, that is, the body-disc height ratio using the Kruskal-Wallis test, instability using Fisher's exact test and clinical outcomes using the chi-square test.

\section{Results}

\section{An analysis of the study patients}

The mean age of the patients was $55.8 \pm 9.7$ years (range, 35 to 70 years) in the spondylolytic spondylolisthesis group, $65.9 \pm 7.1$ years (range, 50 to 78 years) in the degenerative lumbar spondylolisthesis group and $60.4 \pm$ 10.8 years (range, 29 to 75 years) in the degenerative lumbar spondylolisthesis group. The degenerative lumbar spondylolisthesis group showed a significance difference ( $p$ $<0.01)$ of age as compared to that of the other groups. The average follow-up period was $44.7 \pm 18.2$ months (range, 24 to 92 months), $43.2 \pm 18.7$ months (range, 24 to 100

Table 1. Brodsky's criteria

\begin{tabular}{ll}
\hline \hline Desgination & Criterion \\
\hline Excellent & No pain \\
Good & Occasional back or leg pain \\
& No change of work \\
& No change of leisure activity \\
Fair & Frequent back or leg pain \\
& Some change of work \\
& Some change of leisure activity \\
Poor & Disabling pain \\
& Long-term medication \\
& Unable to work \\
\hline
\end{tabular}

Table 2. Summary of cases

\begin{tabular}{|c|c|c|c|c|}
\hline & Spondylolytic spondylolisthesis & Degenerative spondylolisthesis & Spinal stenosis & $p$-value \\
\hline Case (n) & 17 & 22 & 24 & - \\
\hline Age (yr) & $55.8 \pm 9.7$ & $65.9 \pm 7.1$ & $60.4 \pm 10.8$ & $<0.01^{\mathrm{a}}$ \\
\hline Sex (male: female) & $3: 14$ & $1: 21$ & $10: 14$ & $0.02^{\mathrm{b}}$ \\
\hline Follow up (mo) & $44.7 \pm 18.2$ & $43.2 \pm 18.7$ & $42.1 \pm 19.5$ & $0.90^{\mathrm{b}}$ \\
\hline \multicolumn{5}{|l|}{ Fusion level } \\
\hline L3-4 & 0 & 2 & 0 & - \\
\hline L4-5 & 8 & 17 & 22 & - \\
\hline L5-S1 & 9 & 3 & 2 & $<0.01$ \\
\hline
\end{tabular}

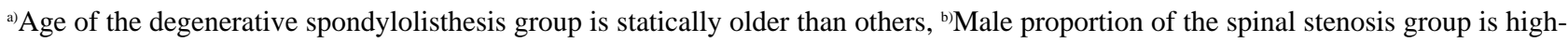
er than others. 
months), and $42.1 \pm 19.5$ months (range, 24 to 102 months), respectively. The differences did not reach statistical significance $(p=0.90)$. In regard to the male-to-female ratio in each group, all three groups showed a female predilection. Yet in the spinal stenosis group, the male-tofemale ratio was found to be significantly higher as compared with that of the other groups $(p=0.02$, the male-tofemale ratios were $3: 14,1: 21$, and $10: 14$ in the corresponding order). In regard to the difference in the fusion segment, the frequency of the L5-S1 level was found to be significantly higher in the spondylolytic spondylolisthesis group $(p<0.01)$ (Table 2). The preoperative degree of anterior listhesis was classified using the Myerding grade, and it was measured as grade 2 in one case of the spondylolytic spondylolisthesis group and grade 1 in the remaining 16 cases of the spondylolytic spondylolisthesis group and all the cases of the degenerative spondylolisthesis group.

\section{An analysis of the radiological outcomes}

\section{(1) A comparison between the intraobserver and inter- observer variations}

A comparison between the intraobserver and interobserver variations for the measurements of the superior adjacent segment's preoperative body-disc height ratio/last bodydisc height ratio and the segmental sagittal angles at the last follow up was done using Spearman correlation analysis. In regard to (the preoperative body-disc height ratio)/(the last body-disc height ratio) and depending on the time point of the measurement, the correlation coefficient $(r)$ was 0.75 and the significance level $(p)$ was 0.01 , which shows a significant correlation. In regard to the segmental sagittal angle measured at a last follow-up, the correlation coefficient $(r)$ and the significance level $(p)$ were found to be 0.89 and 0.01 , respectively, which also showed a high degree of significant correlation.

Interobserver comparison was done by calculating the average value of two measurements, and the interobserver measurement data was also reliable.

\section{(2) The degenerative changes at the superior adjacent disc}

To rule out measurement error due to the magnification and shrinkage of the radiographic images, a comparison was made for the decrement in the intervetebral disc height based on the ratio of the intervertebral disc height vs. the height of the superior vertebral body.
The preoperative body-disc height ratio was $0.43 \pm 0.07$ in the spondylolytic spondylolisthesis group, $0.34 \pm 0.07$ in the degenerative spondylolisthesis group and $0.35 \pm$ 0.06 in the spinal stenosis group, and there was a statistical significant difference $(p<0.01)$ in the spondylolytic spondylolisthesis group. At a last follow-up, the last bodydisc height ratio was also found to be significantly higher in the spondylolytic spondylolisthesis group than that in the other two groups $(p<0.01)$.

In regard to the changes of intervetebral disc height seen in each group, the preoperative body-disc height ratio was $0.43 \pm 0.07$ in the spondylolytic spondylolisthesis group, but it was significantly decreased to $0.40 \pm 0.07$ at a last follow-up $(p<0.01)$. Yet in the degenerative spondylolisthesis group and the spinal stenosis group, the amount of decrease of the body-disc height ratio was not statistically significant $(0.35 \pm 0.07$ to $0.34 \pm 0.06[p=0.49]$ and 0.35 \pm 0.06 to $0.33 \pm 0.08$ [ $p=0.07$ ], respectively).

On comparing the decrement in the intervetebral disc height between the three groups, there was no statistically significant difference $(p=0.45)$.

Preoperatively and at a last follow-up, the segmental sagittal angle of the superior adjacent disc was found to be $4.49 \pm 3.09$ and $5.56 \pm 4.84$, respectively, in the spondylolytic spondylolisthesis group, $4.29 \pm 2.60$ and $3.37 \pm$ 3.40 , respectively, in the degenerative spondylolisthesis group and $4.88 \pm 3.09$ and $2.77 \pm 3.57$, respectively, in the spinal stenosis group. These results showed no significant difference in the segmental sagittal angle of the superior adjacent disc between each groups at the preoperatively $(p=0.77)$ and final follow-up $(p=0.06)$.

In regard to the proportion of cases in which the segmental sagittal angle was measured as $>10$ at a final follow-up and this indicates hypermobility and the proportion of cases in which anterior or posterior listhesis occurred, there were five cases $(27.8 \%)$ and four cases $(22.2 \%)$, respectively, in the spondylolytic spondylolisthesis group, one case (4.3\%) and three cases (13.0\%), respectively, in the degenerative spondylolisthesis group and three cases (11.1\%) and no cases $(0 \%)$, respectively, in the spinal stenosis group. In regard to the proportion of cases in which there was hypermobility or listhesis occurred and this indicates instability, there were five cases (33.3\%), four cases (17.4\%) and three cases $(11.1 \%)$, respectively, in the corresponding order of groups. In the degenerative spondylolisthesis group and the spinal stenosis group, instability was found in less than five cases. Therefore, there is a limitation to make a synchro- 
Table 3. Summary of results

\begin{tabular}{|c|c|c|c|c|}
\hline & Spondylolytic spondylolisthesis & Degenerative spondylolisthesis & Spinal stenosis & p-value \\
\hline Preoperative disc-body ratio & $0.43 \pm 0.07$ & $0.35 \pm 0.07$ & $0.35 \pm 0.06$ & $<0.01^{\text {a) }}$ \\
\hline Follow up disc-body ratio & $0.40 \pm 0.07$ & $0.34 \pm 0.06$ & $0.33 \pm 0.08$ & $<0.01^{\text {a) }}$ \\
\hline Preoperative/Follow up ratio & $1.06 \pm 0.09$ & $1.02 \pm 0.14$ & $1.11 \pm 0.28$ & 0.31 \\
\hline \multicolumn{5}{|l|}{ Segmental sagittal angle $\left(^{\circ}\right)$} \\
\hline Preoperative & $4.49 \pm 3.09$ & $4.29 \pm 2.60$ & $4.88 \pm 3.09$ & 0.77 \\
\hline Follow up & $5.56 \pm 4.84$ & $3.37 \pm 3.40$ & $2.77 \pm 3.57$ & 0.06 \\
\hline \multirow[t]{2}{*}{ Instability } & $6(33.3)$ & $4(17.4)$ & $3(11.1)$ & $0.29^{\mathrm{b})}$ \\
\hline & & & & $0.13^{\mathrm{c})}$ \\
\hline Hypermobility case & $5(27.8)$ & $1(4.3)$ & $3(11.1)$ & - \\
\hline Listhesis & $4(22.2)$ & $3(13)$ & $0(0)$ & - \\
\hline Clinical results & & & & 0.95 \\
\hline Excellent & 5 & 4 & 4 & \\
\hline Good & 7 & 14 & 12 & \\
\hline Fair & 3 & 5 & 4 & \\
\hline Poor & 2 & 1 & 2 & \\
\hline
\end{tabular}

Values are presented as number $(\%)$.

${ }^{a}$ Ratio of the spondylolytic spondylolisthesis group is higher than others, ${ }^{\text {b) }}$ Comparison between the spondylolytic spondylolisthesis group and the degenerative spondylolisthesis group, ${ }^{\circ}$ Comparison between the spondylolytic spondylolisthesis group and the spinal stenosis group.

nous comparison between the three groups. Accordingly, Fisher's exact test was performed between the spondylolytic spondylolisthesis group and the degenerative spondylolisthesis group, and between the spondylolytic spondylolisthesis group and the spinal stenosis group. But there was no statistical significance for each comparison $(p=0.46$ and $p$ $=0.24$, respectively) (Table 3 ).

\section{An analysis of the clinical outcomes}

(1) An analysis of the concordance between the intraobserver and interobserver variations

For analyzing the concordance between the intraobserver and interobserver variations, we measured the kappa value. According to the measurement, the intraobserver kappa value was 0.680 and the interobserver kappa value was 0.620 . These results indicate that there was a significant concordance in the intraobserver and interobserver variations.

\section{(2) Clinical outcomes}

At the last follow-up and based on the Brodsky classification, excellent and good treatment outcomes were seen in 12 cases (70.6\%) of the spondylolytic spondylolisthesis group, in 18 cases $(72.7 \%)$ of the degenerative spondylolisthesis group and in 16 cases (75\%) of the spinal stenosis group. On comparing the clinical outcomes between the groups using chi-square tests, there were no statistically significant differences $(p=0.951)$.

\section{Discussion}

Although various methods of the surgical treatment for spondylolytic spondylolisthesis are used, there has been no surgical treatment established as a superior option than others. The major purposes of surgical treatment include the stabilization of an unstable segment and the decompression of neural structures [16]. Thomsen et al. [17] performed pedicle screw fixation and posterolateral fusion and they obtained a higher fusion rate and satisfactory reduction. However, according to Suk et al. [18], because posterolateral fusion has a higher degree of a loss of the reduction as well as non-union, a higher fusion rate and good clinical outcomes were obtained following a 360 circumferential fusion. Good clinical outcomes were also reported by to the previous studies in which PLIF was performed [19].

As advances have been made for the methods of obtaining solid bone fusion and the follow-up period has been prolonged, solid fusion has been reported to accelerate the degenerative changes of the adjacent segment. There is increased interest in that issue and now studies are actively progressing $[1,4,7,8,20-23]$. There are also opinions that the degenerative change of the adjacent segment of the fusion level is not a complication due to the fusion, but rather it is 
part of the normal aging or degenerative process [6$8,22,23]$. But in a biomechanical study, there were such findings as the increased tension in the segment adjacent to the fusion level, hypermobility and increased intervertebral disc pressure $[4,5]$ and these factors might affect the occurrence of degenerative changes.

There is a variability for the incidence rate of degenerative change in the adjacent segment following lumbar fusion depending on the studies, and the rate of degenerative change has been reported to be approximately $24-49 \%$ $[10,20,24,25]$. Most of the studies have reported that degenerative change occurred in the proximal segment to the fusion level $[7,26]$. Accordingly, in this study, the superior segment to the fusion level was solely examined. There is still a controversy as to the relationship between the degenerative change and the clinical symptoms. For this reason, Hilibrand and Robbins [21] classified it as an adjacent segment disease only when there were suitable clinical symptoms with the appropriate radiologic findings. The proportion of patients who are in need of revision surgery due to the adjacent segment disease is significantly lower than that for the patients who present with degenerative change. In patients who underwent PLIF, Lin et al. [1] noted that a revision surgery was needed in only $2 \%(9 / 465)$ of the total cases at a 1- to 10-year follow-up.

The risk factors reported to be associated with the degenerative changes of the adjacent segment include the patientrelated factors (age, gender, and bone mineral density), anatomical factors (the laminar and facet joints), the preoperative status of the intervertebral disc and facet joints, the number of fusion levels, the fusion methods, the use of instrumentation and the alignment in the coronal plane and sagittal plane, and all of these factors are controversial [812]. It has been universally accepted that changes of the adjacent segment frequently occur as time elapses following lumbar fusion [22].

Of the patient related factors, in regard to gender and age, Kumar et al. [27] noted that there was no gender-related difference in the occurrence of degenerative change at the adjacent segment to the fusion level. Aota et al. [24] reported a high incidence rate of instability of the adjacent segment in patients who were aged 55 years or older. Yet in recent years, many studies have reported that there are no age-related changes [8-10]. In this study, because there was a small number of male patients in both the spondylolytic spondylolisthesis group and the degenerative spondylolisthesis group, statistical analysis could not be easily per- formed. In the spinal stenosis group, there was no genderrelated difference in the occurrence of instability of the adjacent segment. Also in regard to age, the average age was significantly higher in the degenerative spondylolisthesis group as compared with that of the other groups. But the occurrence of adjacent segment degeneration showed no significant difference from that of the other groups.

Lai et al. [13] reported the importance of a tension band mechanism by the posterior column structure as a risk factor for adjacent segment degeneration. They also noted that the degenerative changes of the superior adjacent segment would occur in the early stage due to the increased instability in cases in which the insertion sites of the supraspinous ligament and interspinous ligament were removed in the superior adjacent segment by total excision of the spinous processes. In the case of spondylolytic spondylolisthesis, the total lamenectomy and the damage to the supraspinous ligament and interspinous ligament in the superior adjacent segment are unavoidable. According to their statement, it is expected that the degenerative change would occur in the early stage, but in this study, contradictory results were observed, and based on our clinical experience, there was no tendency that the degenerative change occurred in the early stage in patients with spondylolysis.

Unlike what Lai et al. [13] did, we performed PLIF rather than the posterolateral fusion and this supported the anterior region of the vertebral body and it maintained a more stable lumbar lordosis. It is also assumed that anterior fusion reduced the loading that was increasingly exerted to the adjacent segment. Lee and Langrana [5] reported in a biomechanical study that an increased loading was exerted to the disc in the adjacent segment in cases in which posterior fusion was performed, but the increment of the loading was relatively smaller in those patients in which anterior fusion was performed. In addition, Penta et al. [23] conducted a study on the degenerative change of the adjacent segment to the fusion level and as was seen on magnetic resonance image scans. Those authors reported that the degenerative change of the adjacent intervertebral disc was not aggravated in cases in which anterior fusion was performed.

Madan and Boeree [28] reported that more than good clinical outcomes were observed in approximately $70 \%$ of the total cases of spondylolytic spondylolisthesis that were treated with PLIF. Equivalent clinical outcomes were also observed in this current study. Many studies have also compared the clinical outcomes between the cases of spondylolytic spondylolisthesis treated with PLIF and those treated 
with posterolateral fusion $[18,28,29]$. But there are not many studies where the different disease groups were treated with same PLIF, as was done in the current study. According to this current study, when spondylolytic spondylolisthesis, degenerative spondylolisthesis and spinal stenosis were treated with a single-level PLIF, there were no significant differences in the clinical outcomes at the last follow-up between the disease groups. This requires further long-term follow-up studies.

Lehmann et al. [22] conducted a study on 62 patients who underwent posterolateral fusion with 33-years of follow-up. According to them, instability of the superior adjacent segment occurred in $45 \%$ of the total cases and the spinal stenosis was seen in $42 \%$. Based on these findings, they noted that there was a strong correlation between instability of the superior adjacent segment and spinal stenosis, but there was no correlation between the radiologic findings and symptoms. According to Frobin et al. [30], the first sign of intervetebral disc degeneration was the decreased intervertebral disc height and the intervetebral disc height was decreased as the degeneration progressed. They also noted that there was no significant correlation between the intervetebral disc degeneration and the decreased intervertebral disc height. Therefore, the limitations of this current study are that the occurrence of degenerative change was evaluated based only on the changes in the intervertebral disc height and the radiologic findings of instability of the superior adjacent segment. In addition, based on the characteristics of each disease group, such variables as the gender, age and the fusion level were not adjusted for. Also, there was a limitation of not considering the influence due to the occurrence of degenerative change in the preoperation superior adjacent segment.

\section{Conclusions}

For the surgical management of spondylolytic spondylolisthesis, even following the total removal of the posterior ligament complex of the superior adjacent segment as well as performing total laminectomy, there was no significant difference in the degenerative change of the superior adjacent segment as compared to that of performing partial laminectomy and leaving intact the posterior ligament complex of the superior adjacent segment. Based on these findings, it can be inferred that satisfactory treatment outcomes can be obtained without increasing the risk of developing degenerative change of the superior adjacent segment solely with performing single-level PLIF in the cases in which there are no lesions that might cause clinical symptoms in the superior segment where a listhesis is present.

An average follow-up period of 43 months is relatively short for the evaluation of the degenerative change of the adjacent segment. Thus, longer-term follow-up studies on this are needed. The incidence of adjacent segment degeneration may vary in the cases of L5-S1 fusion and those of L3-4 fusion. Further studies will be needed to examine the effects of the amount of decompression depending on the level of fusion by increasing the size of the population assigned to each disease group.

\section{REFERENCES}

1. Lin PM, Cautilli RA, Joyce MF. Posterior lumbar interbody fusion. Clin Orthop Relat Res 1983;(180):154-68.

2. Bridwell KH, Sedgewick TA, O'Brien MF, Lenke LG, Baldus $\mathrm{C}$. The role of fusion and instrumentation in the treatment of degenerative spondylolisthesis with spinal stenosis. J Spinal Disord 1993;6:461-72.

3. West JL 3rd, Bradford DS, Ogilvie JW. Results of spinal arthrodesis with pedicle screw-plate fixation. J Bone Joint Surg Am 1991;73:1179-84.

4. Yang SW, Langrana NA, Lee CK. Biomechanics of lumbosacral spinal fusion in combined compression-torsion loads. Spine (Phila Pa 1976) 1986;11:937-41.

5. Lee CK, Langrana NA. Lumbosacral spinal fusion: a biomechanical study. Spine (Phila Pa 1976) 1984;9:574-81.

6. Boden SD, McCowin PR, Davis DO, Dina TS, Mark AS, Wiesel S. Abnormal magnetic-resonance scans of the cervical spine in asymptomatic subjects: a prospective investigation. J Bone Joint Surg Am 1990;72:1178-84.

7. Miyakoshi N, Abe E, Shimada Y, Okuyama K, Suzuki T, Sato K. Outcome of one-level posterior lumbar interbody fusion for spondylolisthesis and postoperative intervertebral disc degeneration adjacent to the fusion. Spine (Phila Pa 1976) 2000;25:1837-42.

8. Okuda S, Iwasaki M, Miyauchi A, Aono H, Morita M, Yamamoto T. Risk factors for adjacent segment degeneration after PLIF. Spine (Phila Pa 1976) 2004;29:1535-40.

9. Aiki H, Ohwada O, Kobayashi H, et al. Adjacent segment stenosis after lumbar fusion requiring second operation. $\mathrm{J}$ Orthop Sci 2005;10:490-5.

10. Ha KY, Kim YH, Kang KS. Surgery for adjacent segment changes after lumbosacral fusion. J Korean Soc Spine Surg 2002;9:332-40 
11. Okuda S, Oda T, Miyauchi A, et al. Lamina horizontalization and facet tropism as the risk factors for adjacent segment degeneration after PLIF. Spine (Phila Pa 1976) 2008; 33:2754-8.

12. Wiltse LL, Radecki SE, Biel HM, et al. Comparative study of the incidence and severity of degenerative change in the transition zones after instrumented versus noninstrumented fusions of the lumbar spine. J Spinal Disord 1999;12:2733.

13. Lai PL, Chen LH, Niu CC, Fu TS, Chen WJ. Relation between laminectomy and development of adjacent segment instability after lumbar fusion with pedicle fixation. Spine (Phila Pa 1976) 2004;29:2527-32.

14. Hwang DW, Shin JH, Kim BM, Park YS, Lee JH, Kim EH Radiographic changes of adjacent upper segment performed short segmental lumbosacral fusion: Does total laminectomy influence adjacent upper segmental instability? J Korean Soc Spine Surg 2008;15:31-7.

15. Brodsky AE, Hendricks RL, Khalil MA, Darden BV, Brotzman TT. Segmental ("floating") lumbar spine fusions. Spine (Phila Pa 1976) 1989;14:447-50.

16. Kaneda K, Satoh S, Nohara Y, Oguma T. Distraction rod instrumentation with posterolateral fusion in isthmic spondylolisthesis: 53 cases followed for 18-89 months. Spine (Phila Pa 1976) 1985;10:383-9.

17. Thomsen K, Christensen FB, Eiskjaer SP, Hansen ES, Fruensgaard S, Bünger CE. 1997 Volvo Award winner in clinical studies. The effect of pedicle screw instrumentation on functional outcome and fusion rates in posterolateral lumbar spinal fusion: a prospective, randomized clinical study. Spine (Phila Pa 1976) 1997;22:2813-22.

18. Suk SI, Lee CK, Kim WJ, Lee JH, Cho KJ, Kim HG. Adding posterior lumbar interbody fusion to pedicle screw fixation and posterolateral fusion after decompression in spondylolytic spondylolisthesis. Spine (Phila Pa 1976) 1997;22:210-9.

19. Csécsei GI, Klekner AP, Dobai J, Lajgut A, Sikula J. Posterior interbody fusion using laminectomy bone and transpedicular screw fixation in the treatment of lumbar spondylolisthesis. Surg Neurol 2000;53:2-6.
20. Guigui P, Wodecki P, Bizot P, Lambert P, Chaumeil G, Deburge A. Long-term influence of associated arthrodesis on adjacent segments in the treatment of lumbar stenosis: a series of 127 cases with 9-year follow-up. Rev Chir Orthop Reparatrice Appar Mot 2000;86:546-57.

21. Hilibrand AS, Robbins M. Adjacent segment degeneration and adjacent segment disease: the consequences of spinal fusion? Spine J 2004;4(6 Suppl):190S-194S.

22. Lehmann TR, Spratt KF, Tozzi JE, et al. Long-term followup of lower lumbar fusion patients. Spine (Phila Pa 1976) 1987;12:97-104.

23. Penta M, Sandhu A, Fraser RD. Magnetic resonance imaging assessment of disc degeneration 10 years after anterior lumbar interbody fusion. Spine (Phila Pa 1976) 1995;20:743-7.

24. Aota Y, Kumano K, Hirabayashi S. Postfusion instability at the adjacent segments after rigid pedicle screw fixation for degenerative lumbar spinal disorders. J Spinal Disord 1995;8:464-73.

25. Frymoyer JW, Hanley E, Howe J, Kuhlmann D, Matteri R. Disc excision and spine fusion in the management of lumbar disc disease: a minimum ten-year followup. Spine (Phila Pa 1976) 1978;3:1-6.

26. Ghiselli G, Wang JC, Hsu WK, Dawson EG. L5-S1 segment survivorship and clinical outcome analysis after L4L5 isolated fusion. Spine (Phila Pa 1976) 2003;28:1275-80.

27. Kumar MN, Baklanov A, Chopin D. Correlation between sagittal plane changes and adjacent segment degeneration following lumbar spine fusion. Eur Spine J 2001;10:314-9.

28. Madan S, Boeree NR. Outcome of posterior lumbar interbody fusion versus posterolateral fusion for spondylolytic spondylolisthesis. Spine (Phila Pa 1976) 2002;27:1536-42.

29. Jacobs WC, Vreeling A, De Kleuver M. Fusion for lowgrade adult isthmic spondylolisthesis: a systematic review of the literature. Eur Spine J 2006;15:391-402.

30. Frobin W, Brinckmann P, Kramer M, Hartwig E. Height of lumbar discs measured from radiographs compared with degeneration and height classified from MR images. Eur Radiol 2001;11:263-9. 